Journal of Animal and Veterinary Advances 11 (16): 2910-2916, 2012

ISSN: $1680-5593$

(C) Medwell Journals, 2012

\title{
Acute Fibrosarcomas Caused by Avian Leukosis Virus Subgroup J Associated with v-fps Oncogene
}

\author{
Hao Chen, Yi-Xin Wang, Peng Zhao, Jian-Liang Li and Zhi-Zhong Cui \\ Shandong Engineering Research Center for Animal Disease Control and Prevention, \\ College of Animal Science and Technology, Shandong Agricultural University, \\ Tai' an, 271000 Shandong, P.R. China
}

\begin{abstract}
To explore the reason of acute fibrosarcoma formation in chickens, researchers detected the existence of viral antigens in the cytoplasm of infected CEFs by monoclonal antibody against ALV-J and rabbit polyclonal antibody against oncogene-fps protein. PCR amplified the whole genome sequence of the replication-competent ALV-J (SD1005), alignment of the complete sequence demonstrated that it has no remarkable mutation in the genome and appears not to be the real pathogen inducing the acute tumorigensis. Additionally, we obtained several genome sequences associated with ALV-J (Fu-J1-6) which is defective with parts of gag, pol and env genes replaced by a sequence of fps gene. The sequences of Fu-Js revealed that their genomes are closely related to that of SD1005 and probably derived from it in accordance with the high homology $(>99 \%)$ in env gene between them. Notably, compared with homologous region of Fujinami and PRCII, the obtained v-fps genes in the study have two deletion mutations at both ends with the major diversity at the 3'-fps end, especially. So, researchers infer that it seems to be unstable for junction site in the process of recombination between c-fps oncogene and retrovirus ALV, a majority of variable individuals at gene level (quasispecies) will generate, one strain of which has biological activity at least. Obviously, the induction of acute fibrosarcomas in the experiment occurs through mechanisms involving the direct activation of viral oncogene $\mathrm{v}$-fps in Fu-Js rather than insertional activation of cellular oncogene $\mathrm{c}$-fps, revealing that the tumorigenesis induced by ALV-J is probable to involve fps oncogene besides for the myc oncogene. This is the first report on acute fibrosarcomas caused by ALV-J containing v-fps oncogene since the identification of this virus in 1988.
\end{abstract}

$\underline{\text { Key words: Avian leukosis virus subgroup J, fibrosarcoma, acute tumorigenesis, fps oncogene, quasispecies }}$

\section{INTRODUCTION}

Avian Leukosis/Sarcoma Viruses (AL/SVs) had been divided into non-acute transforming virus and acute transforming virus in accordance with different oncogenesis mechanisms. Non-acute transforming virus caused tumors after a long latent period of $>90$ days by insertional activation of cellular proto-oncogene while acute transforming virus could induce a fast tumorigenesis within several weeks through direct transcription of viral oncogene (Fadly and Nair, 2008). The HPRS-103 strain, prototype of ALV-J has a genome without any viral oncogene neither cause tumors rapidly in vivo and nor transform myeloid cell in vitro (Payne et al., 1992).

However, acutely transforming viruses with v-myc oncogene had been isolated from cases of myelocytomatosis induced experimentally by HPRS-103
(Payne et al., 1993) most of them can transform cultured chicken bone marrow cells in vitro and induce a variety of acute tumors including myelocytomatosis, nephroblastoma and renal adenomas/adenocarcinomas in chickens. Lately, acutely transforming ALV-J strains with $\mathrm{v}$-myc have also been isolated from naturally infected parent flocks (Venugopal et al., 2000) although, they mainly induced erythroblastosis as well as myelocytomatosis.

Fujinami Sarcoma Virus (FSV) was discovered in Japan (Fujinami and Inamoto, 1914). Previous studies have shown that it has a genome with v-fps oncogene which is different from v-src in Rous Sarcoma Virus (RSV) although, both of them can cause acute fibrosarcomas in chickens (Shibuya and Hanafusa, 1982). PRCII is a naturally obtained $5^{\prime}$-fps deletion of 1020 nucleotides mutant showed low efficiency of transforming activity on cells and decreased carcinogenicity in chickens

Corresponding Author: Zhi-Zhong Cui, College of Animal Science and Technology, Shandong Agricultural University, Tai'an, 271000 Shandong, P.R. China 
(Carlberg et al., 1984). Over the past few years, we have observed the occurrence of lesions indicative of fibrosarcoma in tissues from parent layers and hybrid chickens (cross-breeding between commercial female layers and white meat-type male) in China.

Researchers in the lab have made a definite diagnosis of acute fibrosarcoma in these chickens which is characterized by rapid growth and infiltration into surrounding tissues and metastasis and demonstrated that it is associated with the infection of ALV-J (Liu et al., 2011; Li et al., 2012). The study was designed to probe the true pathogen which resulted in acute tumorigenesis from the perspectives of immunology and molecular biology.

\section{MATERIALS AND METHODS}

Identification of viruses: Sarcoma samples were collected from infected chickens. A 10\% tissue homogenates suspensions in Phosphate-Buffered Saline (PBS) were prepared and $0.2 \mathrm{~mL}$ of the filtered supernatant was inoculated into monolayer cultures of Chicken Embryo Fibroblast (CEF) (from SPAFAS SpecificPathogen-Free chickens). The CEF cultures were examined for development of Cytopathic Effect (CPE) daily for 9 days then collected as the first passage cell virus stock for use of chicken inoculation experiment.

The Indirect Fluorescence Assay (IFA) was performed in parallel CEFs cultured in microscope slides with a anti-ALV-J-specific Monoclonal Antibody (Mab) JE9 (Qin et al., 2001) and a anti-fps rabbit polyclonal serum as the primary antibody for the detection of ALV-gp85 and v-fps antigen, respectively. The Mab JE9 was conserved in the lab which is specific for Avian leukosis virus subgroup J. The anti-fps polyclonal antibody was prepared in the lab which is specific for the purified fps proteins expressed from Escherichia coli (E. coli).
Goat-anti-mouse and goat-anti-rabbit monoclonal antibody (second antibody) labeled with FITC was supplied by sigma corporation.

Chicken inoculation: The suspensions of the first passage cell virus stocks were quantified by measuring the $50 \%$ Tissue-Culture Infective Dose $\left(\mathrm{TCID}_{50}\right)$ of ALV-J. The 2 weeks old SPF chickens (from Jinan SPAFAS Poultry Company Ltd. Jinan, China) were inoculated neck-subcutaneously with the suspensions of the first passage cell virus stocks $\left(10^{4}\right.$ unit $\mathrm{TCID}_{50}$ each bird). Chickens in negtive control group were inoculated similarly with uninfected CEFs. All the chickens (10 birds each group) were maintained separately in two isolation room in experimental animal house facilities at Shandong Agricultural University, Tai' an, China. Researchers will observe the occurrence of tumor and measure the sizes of sarcomas every 3 days.

Amplification of genes: The viral RNA was extracted from sarcoma tissues by using EZNA ${ }^{\mathrm{TM}}$ Viral RNA kit (Omega, Norcross, GA, USA) according to the manufacture's instruction. The specific primers for ALV-J and v-fps chimera molecules were designed (Table 1) and synthetized for the amplification of genes of replication competent viruses and defective viruses, respectivly. RT reaction was carried out with TaKaRa RNA PCR kit (AMV) Ver. 3.0 (TaKaRa, Dalian, China) in accordance with the supplied protocols.

All PCRs were performed in mastercycler ep gradient (Eppendorf, Hamburg, Germany) using the following parameters: $94^{\circ} \mathrm{C}$ initial denaturation for $7 \mathrm{~min}, 32$ cycles of $94^{\circ} \mathrm{C}$ initial denaturing for $40 \mathrm{sec}, 59-62^{\circ} \mathrm{C}$ annealing for $40 \mathrm{sec}$ and $72^{\circ} \mathrm{C}$ extension for $1-3 \mathrm{~min}$ followed by a $8 \mathrm{~min}$ extension at $72^{\circ} \mathrm{C}$. PCR products were purified from agarose gel using a QIAquick Gel Extraction kit (Qiagen, Hilden, Germany) and sequenced with Automated Laser Fluorescence DNA Sequencer (ABI). The sequences were analyzed by DNAStar program (Madison, WI, USA).

Table 1: Specific primers used in this study

\begin{tabular}{|c|c|c|c|c|}
\hline Primer names & Sequence $\left(5^{\prime}-3^{\prime}\right)$ & Amplified fragment or gene & Melting temperature $\left({ }^{\circ} \mathrm{C}\right)$ & Extension time (sec) \\
\hline gag-F & CACCACATTGGTGTGCACCTGGGT & J-gag & 62.0 & 3.0 \\
\hline gag- $R$ & GAAGGGGCCACTGGTCAATCCACA & & & \\
\hline pol-F & GAGATTGTCTGCAGGGCCTAGGGCT & J-pol & 62.0 & 3.0 \\
\hline pol-R & TGGCAGCAAGGGTGTCTTCTCCG & & & \\
\hline env-F & GAGGTGACTAAGAAAGATGAGGCGA & J-env & 62.0 & 2.0 \\
\hline env-R & САTCTCССССТCCСТATGCGAAAGC & & & \\
\hline 3'UTR-F & GGCTTCGGTTGTACGCGGATAGGA & $\mathrm{J}$-Circle & 59.0 & 1.0 \\
\hline 5'UTR-R & CTTCCAACGACCCTCTGAGTGCTCG & & & \\
\hline 5 R-F & GCCATTTTACCTCCCACCACATTGG & 5UTR-gag-5'fps & 62.0 & 2.0 \\
\hline 5 'fps-R & CCAGCTGAGAGAACATGTGGTGCAG & & & \\
\hline gag-F & CCCCATATGCCTTATGGATGGACGC & gag- 3 'fps & 62.5 & 2.5 \\
\hline 3'fps-R & CCTGTGGATGCAGTGCTTGCTTTCC & & & \\
\hline 3'fps-F & GCAGCCCATTTACATCGTCATGGAG & 3'fps-3'UTR & 60.0 & 2.0 \\
\hline 3'R-R & TGTGGTGGGAGGTAAAATGGCGTT & & & \\
\hline
\end{tabular}




\section{RESULTS}

Results of IFA: The infected CEFs were detected by IFA, the results showed that they were positive to JE9 (Fig. 1a) indicating that ALV-J existed in the fibrosarcoma tissues. We designated this ALV-J isolate as SD1005 (Liu et al., 2011). Besides, the infected CEFs were also shown to be positive to anti-v-fps antibody (Fig. 1b) while the non-infected CEF cells reacted weakly (Fig. 1c) implying that virus antigens also contain fps-coded protein other than ALV-J-encoded structural and functional proteins which is characteristic of Fujinami-like sarcoma virus. Furthermore, the morphology of the CEFs infected by supernatant was similar to that of the negtivecontrol uninfected $\mathrm{CEFs}$ and no $\mathrm{CPE}$ was observed.

Experimental reproduction of the disease: The palpable sarcomas occurred in the neck on the 10-12th days post inoculation at the earliest and expanded to all chickens on the 15 th days, grew rapidly with a diameter of $3.5-4.6 \mathrm{~cm}$ within 10 days after the initial occurrence in the inoculated chickens (Table 2). Postmortem examination showed that some sarcomas emerged as lumps like balls with a diameter of 4-5 cm (Fig. 1d) and had a hard texture. Much of mucus and blood flew occasionally along the crosssection. The duplicated sarcomas were similar with clinical obtained samples and histopathology showed that they were fibrosarcomas too indicating that the experimental reproduction of the disease is successful.

Amplification of the genome of SD1005: The obtained genome sequence of SD1005 is 7633 nt long. With reference to the genome sequences of 12 strains ALV-J, sequences alignment were performed. Its gag gene is $2106 \mathrm{nt}$ long and has a 94.8-98.8\% nucleotide homology with reference isolates; pol gene has $2622 \mathrm{nt}$ and shared $96.8-98.8 \%$ nucleotide identity; env gene is $1707 \mathrm{nt}$ long and has $91.4-97.9 \%$ nucleotide homology with them. It has the most high nucleotide homology with JS09GY6 (Wu et al., 2010) which was isolated from commercial egg-type chickens with myelocytomas and hemangiomas, while shares the relatively low identity in env gene with SD9902 (Duand Cui, 2002), an acutely transforming ALV-J strain inducing myelocytomatosis.

Besides, its rTM fragment is only 35 nt long with a large deletion in the rear section, the same as JS09GY6. As a role of non-functional domain, it appears that this mutation is not associated with the acute fibrosarcomas formation. As far as the whole genome's concerned, the ALV-J in this experiment has a similar structure and a nucleotide homology of $97 \%$ with JS09GY6. Compared with HPRS-103, the common characterization shared by
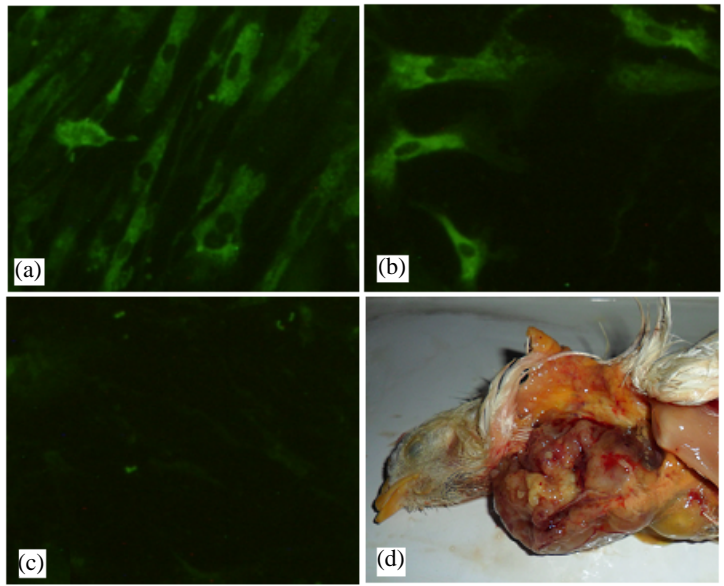

Fig. 1: The results of IFA and animal regression test. a) Inspection of viral envelope protein in inoculated CEF by IFA with JE9. The fluorescence was located in cytoplasm. Original magnification $\mathrm{x} 100$; b) Detection of potential fps protein in inoculated CEF by IFA with anti-fps rabbit serum. The fluorescence was located in cytoplasm. Original magnification $\mathrm{x} 100$; c) Detection of uninfected CEF by IFA with anti-fps rabbit serum. Original magnification $\mathrm{x} 100$ and d) Sarcoma lumps observed in a SPF chicken on day 18 after inoculation subcutaneously at 2 weeks of age with the suspension of the $\mathrm{CEF}$ inoculated by filtered supernatant of sarcoma sample

Table 2: Tumor formation in vivo by inoculated CEFs suspension Tumor size $(\mathrm{mm})^{\mathrm{a}}$ at indicated day after innoculation

\begin{tabular}{llrrrrr} 
Groups & 9 & 12 & 15 & 18 & 21 & 30 \\
\hline Infected group & 0 & 8 & 18 & 30 & 35 & $\mathrm{D}^{\mathrm{b}}$ \\
Negtive control & 0 & 0 & 0 & 0 & 0 & 0 \\
\hline
\end{tabular}

${ }^{a}$ Tumor size represents the average tumor diameter in live chickens inoculated subcutaneously in the neck; ${ }^{b} \mathrm{D}$ : Dead

them shows the existence of two deletion mutations in their genomes. One is the deletion of an amino acid (Lys, at the 179th site in the gp85 protein of HPRS103) in the protein encoded by the hrl region.

The other is the deletion of single-base $\mathrm{C}$ in $\mathrm{E}$ element (at the 30th site in E of HPRS-103), it creats a distinct binding site for c-Ets-1(p54) which is associated with vascular endothelial cell differentiation (Dorsey et al., 2002). However, it seemed farfetched to consider that these differences could result in the acute tumorigenesis duing to the property of JS09GY6 causing non-acute hemangiomas. Alignment of genes or fragments and nucleotide homology analysis between SD1005 and other ALV-J strains (Table 3) showed that the ALV-J isolate in the study has no important or fatal mutations in its 
Table 3: Nucleotide homology of SD1005 with other ALV-J reference strains

\begin{tabular}{|c|c|c|c|c|c|c|c|c|}
\hline \multirow[b]{2}{*}{ ALV-J strains name } & \multicolumn{8}{|c|}{ Genes or fragments of SD1005 } \\
\hline & LTR 325nt & gag $2106 \mathrm{nt}$ & pol 2622nt & env $1707 \mathrm{nt}$ & Ins.TM $220 \mathrm{nt}$ & $\mathrm{rTM}^{\mathrm{D}} 35 \mathrm{nt}$ & DR1 93nt & E (XSR)146nt \\
\hline HPRS-103 (1995) & 90.5 & 95.6 & 97.5 & 93.8 & 93.2 & 97.1 & 96.8 & 93.2 \\
\hline YZ9902 (1999) & 90.5 & 95.5 & 97.3 & 94.3 & 92.7 & 88.6 & 89.2 & $\mathrm{D}^{c}$ \\
\hline ADOL-7501 (2001) & 90.2 & 96.1 & 98.0 & 91.4 & 94.1 & 65.7 & 92.5 & 94.5 \\
\hline NX0101 (2001) & 90.5 & 98.8 & 98.5 & 93.6 & 94.1 & 77.1 & 88.2 & $\mathrm{D}$ \\
\hline NM2002-1 (2002) & 91.4 & 95.8 & 97.2 & 93.8 & 94.5 & 77.1 & 88.2 & $\mathrm{D}$ \\
\hline JS-nt (2003) & 91.4 & 96.2 & 97.1 & 94.0 & 94.5 & 77.1 & 89.2 & $\mathrm{D}$ \\
\hline SD07LK1 (2007) & 88.6 & 97.1 & 98.8 & 94.2 & 92.3 & 74.3 & 92.5 & 88.4 \\
\hline $\mathrm{NHH}(2007)$ & 90.4 & 95.4 & 96.8 & 94.0 & 90.5 & 54.3 & 97.8 & 91.8 \\
\hline HAY013 (2008) & 89.2 & 95.5 & 97.3 & 94.5 & 94.5 & 77.1 & 88.2 & $\mathrm{D}$ \\
\hline SCDY1 (2009) & 87.9 & 94.8 & 98.1 & 94.0 & 90.9 & 45.7 & 90.3 & $\mathrm{D}$ \\
\hline JS09GY6 (2009) & 96.3 & 96.2 & 98.1 & 97.9 & 99.1 & 100.0 & 96.8 & 95.9 \\
\hline SD9902 (1999) & $92.5^{b}$ & - & - & 95.2 & 95.0 & 77.1 & 88.2 & $\mathrm{D}$ \\
\hline
\end{tabular}

${ }^{\mathrm{a}}$ Represents the isolated year, ${ }^{\mathrm{b}}$ Represents there is no related sequence in available and ${ }^{\mathrm{C}} \mathrm{D}$ means that the alignment of nucleotide homology is not performed due to deletion mutations of corresponding fragment

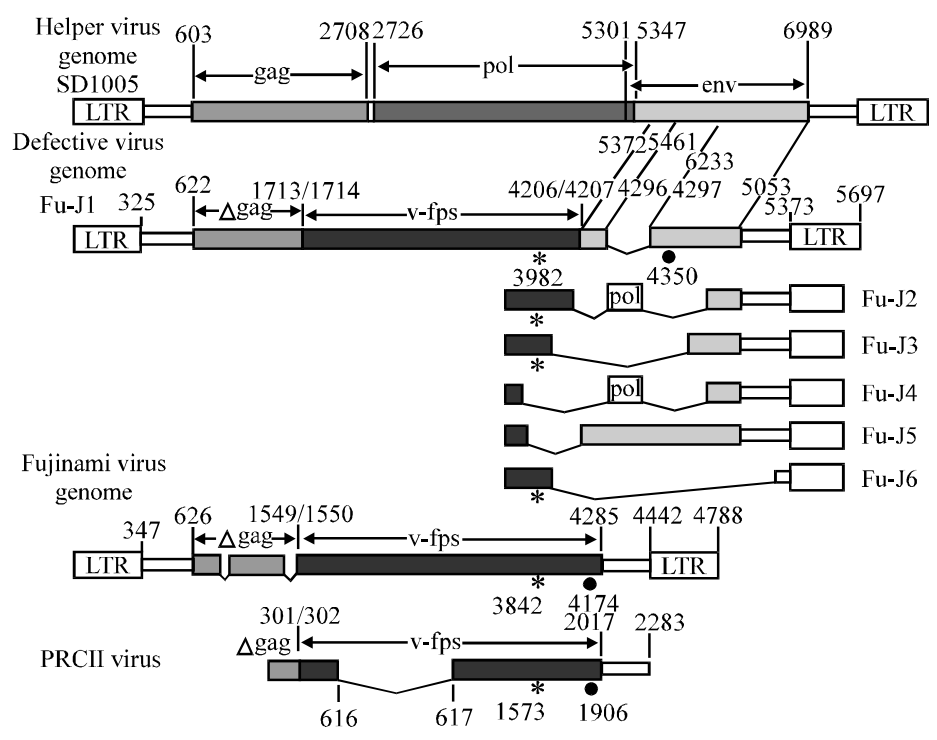

Fig. 2: Comparisons of the $v-f p s$ gene and its environs in the genome of ASV-FJ1, FSV and PRCII. Asterisk indicates canonical site for phosphorylated tyrosine dot represents terminator of the long open reading frame in the gag-fps hybrid gene

genome structure which could result in the change of pathogenicity and tumorigenesis pedigree when compared with previous ALV-Js

\section{Genome structure of the defective virus associated} with v-fps: Three sections of overlapped chimera molecules were amplified by use of primers specific to the oncogene fps while no any other specific fragment was obtained using primers for other oncogenes, src, fps, ros, yes and myc. The obtained chimeras were 5'UTR-gag5'fps (1781 bp), gag-3'fps (2363 bp) and 3'fps-env-3'UTR (1886 bp), respectively. The nucleotide homologies of overlay regions are between 99.8 and $100 \%$ indicating that they come from the same genome. The whole proviral genome of defective virus was obtained through the connection of the upper three sections with both obtained LTRS. We name it as Fu-J1. Its proviral genome structure was 5 'LTR- $\Delta$ gag-fps- $\Delta$ env-3'LTR as shown in Fig. 2. Its fps gene shared $98.5 \%$ nucleotide homology with homologous sequence of Fujinami and PRCII.

The $\Delta e n v$ gene sequence in the chimera molecule had $99.8 \%$ nucleotide identity with the env gene of SD1005 from the same tumor sample showing that the right ALV-J strain is not only the helper virus but also the possible progenitor of the defective virus. Different from Fujinami and PRCII, the fps gene of Fu-J1 had two mutagenesis at two ends: one was the deletion of 21 nucleotides at the initiation of $5^{\prime}$-fps portion which induces the absence of 7 amino acid residues (ASGQLHR); the other was substitution of 108 nucleotides at the end of 3 '-fps portion 


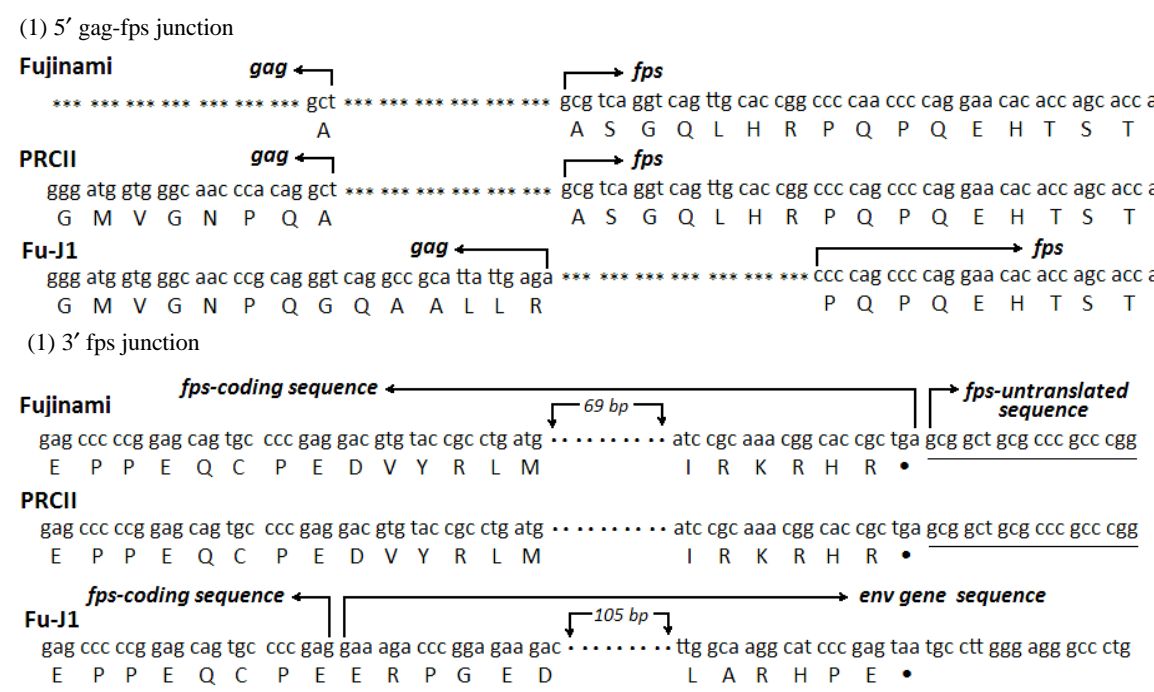

Fig. 3: The leftward and rightward junction sites in the v-fps of Fujinami, PRCII and Fu-J1. Asterisk indicates base deletion; dot means terminator of the ORF in the gag-fps hybrid gene ellipsis represents elliptical nucleotides due to space limitation

with 35 fps-encoded aa replaced by 47 env-encoded aa (Fig. 2 and 3). So, different to FSV and PRCII, its leftward and rightward junctions with $A L V$ genes manifested significant divergence (Fig. 3).

At the left joint site, its $\Delta$ gag gene was longer and encoded a more amino acid residues QAALLRP, amino acid residue $\mathrm{P}$ was owned by both of gag and fps thus it seemed probable that the nucleotides sequence of ccc encoding amino acid residue $\mathrm{P}$ was the leftward recombinational site; at the rightward joint site, the stop codon was in the env gene which caused the front part of $\Delta e n v$ gene was in the ORF however its encoded amino acid sequence was not part of envelope protein. These characteristics were not found in Fujinami and PRCII. It was worthy to mention that Fu-J1-fps had a sequence of 1020 nucleotides which was absent in PRCII although, its deduced amino acid sequence shared higher homology with PRCII than FSV.

\section{Diversity of the carboxy terminus of the v-fps product:} Except for Fu-J1, several distinct PCR products of the rear portion of chimeras (FJ2-6) were obtained and presented diagrammatically together in Fig. 2. In their clones, 3'-fps portion was shorter than that of Fu-J1, especially the clones of Fu-J4 and Fu-J5 even lacked amino acid residue Tyr. Additionally, chimeras contained part of pol gene in Fu-J2 and Fu-J4 whereas no env gene existed in Fu-J6. However, the env part in these molecules all shared over 99\% identities in nucleotide level with the env gene without exception.

\section{DISCUSSION}

The tumor disease associated with ALV-J had since its description $>10$ years ago in China (Du et al., 2000) been prevalent in many areas (Xu et al., 2004; Cheng et al., 2005; Sun and Cui, 2007; Wang and Cui, 2008; Gao et al., 2010). Non-acute myelocytoma/ lymphocytoma/hemangioma were observed in these researches however, acute tumorigenesis caused by ALV-J has not been found here till now. But researchers in UK (Payne et al., 1993; Venugopal et al., 2000) had isolated several strains of acutely transforming virus associated with ALV-J and identified that acute myelocytomatosis and erythroblastosis induced by them were related to the direct activation of viral oncogene myc in 1993 and 2000, respectively indicating that highly oncogenic ALV-J, capable of inducing a different spectrum disease other than the widely reported myelocytomatosis could be established in naturally infected flocks.

Meanwhile many previous studies showed that the viruses of acute sarcoma-inducing were mainly Rous Sarcoma Virus (RSV), Fujinami Sarcoma Virus (FSV) (Lee et al., 1980), UR2 Sarcoma Virus (UR2SV) (Neckameyer and Wang, 1985) and Y73 Sarcoma Virus (Y73SV) (Kitamura et al., 1982) which contained different viral oncogenes such as src, fps, ros, yes. It was worthy to note that their associated helper viruses belonged to ALVs-A/B other than ALV-J because ALV-J was not discovered and identified until 1988. Acute fibrosarcomas were diagnosed in filed cases and established in experimental infections of chickens by inoculation with 
the extract of field tumor samples (Liu et al., 2011; Li et al., 2012) and isolated ALV-J associated virus. The plasmas in the inoculation site emerged on the 10-12th days post inoculation and grew fast with infiltration into the surrounding tissues while the acute transforming virus with v-myc 966 strain took at least 55 days for myelocytomas development (Chesters et al., 2001). The detection for viral antigen by IFA demonstrated ALV-J and some kind of Avian Sarcoma Virus (ASV) with fps oncogene exist in the cytoplasm of positive infected CEFs. Moreover, the genomes of SD1005 and Fu-J1 were successful to be amplified confirming the presence of the foregoing two viruses. According to the molecular mechanism of tumorigenesis induced by Avian Leukosis/Sarcoma Viruses (AL/SVs), it is inferred that the defective virus Fu-J1 might be responsible for the acute fibrosarcomas outbreaks in the field and experimental cases by the direct activation of $\mathrm{v}$-fps oncogene while the replication-competent ALV-J (SD1005) just play a secondary role as helper virus.

But there remains one problem that Fu-J1 appears to be only one strain of quasispecies group of defective $\mathrm{ALV}$ which derive from the frequent recombination events between the ALV genome and c-fps oncogene. Because several other sequences of rear portion chimeras were also, obtained and showed a fps-env region closely resembling that of Fu-J1 virus although, the recombination junction and the length of fps or env region varied among the individual PCR products. Not alone, this similar phenomenon was also reported in the research of 966 virus: different PCR products of gag-5'myc junction domain were obtained. So, researchers infer that the emergence of quasispecies group may be an universal phenomenon in the process of acute transforming $A L V$ formation.

FSV and PRCII sarcoma virus are two strains of replication-defective avian sarcoma viruses which can induce acute sarcomas in vivo and transformation of fibroblasts in vitro (Breitman et al., 1981). The pathogenicity has been attributed to a single viral oncogene v-fps (Pawson et al., 1980; Hanafusa et al., 1981). In their genomes, $v$-fps is fused to a portion of the viral gene gag which encodes the internal structural proteins of the virus. The hybrid gene gives rise to a fusion protein which displays a tyrosine-specific protein kinase activity that can phosphorylate tyrosine in protein substrates (Feldman et al., 1980).

In the study, the Fu-Js proviral genomes all have a read through hybrid gene, characterized by gag-fps-env or gag-fps some of which even possess amino acid residue Tyr which could be phospholated by phosphotyrosine kinase. So, there should be at least one strain which is responsible for the acute fibrosarcomaformation but it is not clear that which strain makes effect in the tumorigenisis so far. Noticeably, the defective viruses identified in this research have no capacity in transforming CEFs in vitro although, it can cause a severe tumorigenesis in chickens. It is presumed that this property may be related with the relatively distinct fps oncogene we amplified but more exploration and research should be done.

\section{CONCLUSION}

In the recent, 2-3 years there have been several other reports on the emergence of fibrosarcomas in China (Chen et al., 2011; Yang et al., 2011) this hints the new evolution of the tumorigenesis pedigree of ALV-J in the area. The findings in this study indicates that the defective virus containing $\mathrm{v}$-fps oncogene may be the true pathogen which is responsible for the acute fibrosarcoma-formation based on the successful amplification of genome and detection of viral antigen. Moreover, it implys that the activation of $\mathrm{v}$-fps oncogene may be another pathway in which ALV-J causes malign tumors in chickens.

\section{ACKNOWLEDGEMENT}

The research was supported by the grant (\#31172330) from National Natural Science Foundation of China.

\section{REFERENCES}

Breitman, M.L., J.C. Neil, C. Moscovici and P.K. Voqt, 1981. The pathogenicity and defectiveness of PRCII: A new type of avian sarcoma virus. Virology, 108: 1-12.

Carlberg, K., M.E. Chamberlin and K. Beemon, 1984. The avian sarcoma virus PRCII lacks 1020 nucleotides of the $f p s$ transforming gene. Virology, 135: 157-167.

Chen, H.B., L.L. Yu, Y.P. Jiang, Y. Wang and F. Wang et al., 2011. Multipal tumors induced by avian leucosis virus subgroup J in layer flock. Acta. Vet. Zoo. Sin., 42: 1491-1496.

Cheng, Z.Q., L. Zhang, S.D. Liu, L.J. Zhang and Z.Z. Cui, 2005. Emerging of avian leukosis virus subgroup J in a flock of Chinese local breed. Acta. Microbiol. Sin., 45: 584-587.

Chesters, P.M., K. Howes, J.C. McKay, L.N. Payne and $\mathrm{K}$. Venugopal, 2001. Acutely transforming avian lekosis virus subgroup J strain 966: Defective genome encodes a 72-Kilodaton gag-myc fusion protein. J. Virol., 75: 4219-4225. 
Dorsey, J.F., J.M. Cunnick, S.M. Mane and J. Wu, 2002. Regulation of the Erk2-Elk1 signaling pathway and megakaryocytic differentiation of Bcr-Abl (+) K562 leukemic cells by Gab2. Blood, 99: 1388-1397.

Du, Y. and Z.Z. Cui, 2002. Study on pathogenicity of Chinese strains of subgroup J avian leukosis viruses. Sci. Agri. Sin., 35: 430-433.

Du, Y., Z.Z. Cui, A. Qin, R. Silva and L. Lee, 2000. Isolation of subgroup $\mathrm{J}$ avian leukosis viruses and their partial sequence comparison. Chin. J. Virol., 16: 341-346.

Fadly, A.M. and V. Nair, 2008. Leukosis/Sarcoma Group. In: Diseases of Poultry, Saif, Y.M., A.M. Fadly, J.R. Glisson, L.R. McDougald, L.K. Nolan and D.E. Swayne (Eds.)., Blackwell Publishing, Ames, pp: 514-568.

Feldman, R.A., T. Hanafusa and H. Hanafusa, 1980. Characterization of protein kinase activity associated with the transforming gene product of Fujinami sarcoma virus. Cell, 22: 757-765.

Fujinami, A. and K. Inamoto, 1914. Ueber geschwulste bei japanischen haushuhnern, insbesondere uber einen transplantablen tumor. Z. Krebsforsch., 14: 94-119.

Gao, Y.L., L.T. Qin, W. Pan, Y.Q. Wang, X. Le Qi, H.L. Gao and X.M. Wang, 2010. Avian leukosis virus subgroup $\mathrm{j}$ in layer chickens. China. Emerg. Infect. Dis., 16: 1637-1638.

Hanafusa, T., P.B. Mathey, R.A. Feldman and H. Hanafusa, 1981. Mutants of Fujinami sarcoma virus which are temperature sensitive for cell transformation and protein kinase activity. J. Virol., 38: 347-355.

Kitamura, N., A. Kitamura, K. Toyoshima, Y. Hirayama and M. Yoshida, 1982. Avian sarcoma virus Y73 genome sequence and structural similarity of its transforming gene product to that of Rous sarcoma virus. Nature, 297: 205-208.

Lee, W.H., K. Bister, A. Pawson, T. Robins, C. Moscovici and P.H. Duesberg, 1980. Fujinami sarcoma virus: An avian RNA tumor virus with a unique transforming gene. Proc. Nat. Acad. Sci. USA., 77: 2018-2022.

Li, C.L., H. Zhang, P. Zhao and Z.Z. Cui, 2012. Establishment of a bird experiment model for chicken acute fibrosarcomas induced by ALV-J associated virus. Sci. Agri. Sin., 45: 548-555.

Liu, S.Q., B. Wang, Z.J. Zhang, J. Wang, S.H. Sun and Z.Z. Cui, 2011. The separation of subgroup A and J ALV in soft tissue sarcomas of 817 broiler hybrids. Acta. Vet. Zoo. Sin., 42: 396-401.
Neckameyer, W.S. and L.H. Wang, 1985. Nucleotide sequence of avian sarcoma virus UR2 and comparison of its transforming gene with other members of the tyrosine protein kinase oncogene family. J. Virol., 53: 879-884.

Pawson, T., J. Guyden, T.H.Kung, K. Radke, T. Gilmore and G.S. Martin, 1980. A strain of Fujinami sarcoma virus which is temperature-sensitive in protein phosphorylation and cellular transformation. Cell, 22: 767-775.

Payne, L.N., A.M. Gillespie and K. Howes, 1992. Myeloid leukaemogenicity and transmission of the HPRS-103 strain of avian leukos is virus. Leukemia, 6: 1167-1176.

Payne, L.N., A.M. Gillespie and K. Howes, 1993. Recovery of acutely transforming viruses from myeloid leukosis induced by the HPRS-103 strain of avian leucosis virus. Avian. Dis., 37: 438-450.

Qin, A.J., Z.Z. Cui, L. Lee and A. Fadly, 2001. Development and characterization of monoclonal antibodies to subgroup J avian leukosis virus. Acta. Vet. Zoo. Sin., 32: 556-562.

Shibuya, M. and H. Hanafusa, 1982. Nucleotide sequence of Fujinami sarcoma virus: Evolutionary relationship of its transforming gene with transforming genes of other sarcoma viruses. Cell, 30: 787-795.

Sun, S.H. and Z.Z. Cui, 2007. Epidemiological and pathological studies of subgroup J avian leukosis virus infections in Chinese local yellow chickens. Avian. Pathol., 36: 221-226.

Venugopal, K., K. Howes, D.M.J. Flannery and L.N. Payne, 2000. Isolation of acutely transforming subgroup $\mathrm{J}$ avian leukosis viruses that induce erythroblastosis and myelocytomatosis. Avian. Pathol., 29: 327-332.

Wang, H. and Z.Z. Cui, 2008. The identification and sequence analysis of $\mathrm{ALV}-\mathrm{J}$ isolated from layers. Chin. J. Virol., 24: 369-375.

Wu, X.P., K. Qian, A.J. Qin, H.Y. Shen, P.P. Wang, W.J. Jin and Y.M. Eltahir, 2010. Recombinant avian leukosis viruses of subgroup $\mathrm{J}$ isolated from field infected commercial layer chickens with hemangioma and myeloid leukosis possess an insertion in the $\mathrm{E}$ element. Vet. Res. Commun., 34: 619-632.

Xu, B., W. Dong, C. Yu, Z. He and Y. Lv et al., 2004. Occurrenceof avian leukosis virus subgroupJ in commercial layer flocks in China. Avian. Pathol., 33: 13-17.

Yang, F., H.L. Sun, W. Ni, G.X. Che and S.D. Liu, 2011. Epidemiological study of avian leukosis on layer chickens with different strains in Shandong province. Acta. Vet. Zoo. Sin., 42: 698-703. 